\title{
Systemic immune-inflammation index predicts the clinical outcome in patients with nasopharyngeal carcinoma: a propensity score-matched analysis
}

\author{
Wenjie Jiang ${ }^{1, *}$, Yuan Chen ${ }^{1, *}$, Jin Huang ${ }^{1}$, Dan $\mathrm{Xi}^{1}$, Jun Chen ${ }^{2}$, Yingjie Shao ${ }^{1}$, \\ Guoping $\mathrm{Xu}^{1}$, Wenming Ying ${ }^{1}$, Jun $\mathrm{Wei}^{1}{ }^{1}$, Junjun Chen ${ }^{1}$, Zhonghua Ning ${ }^{1}$, Wendong \\ $\mathbf{G u}^{1}$ and Honglei $\mathbf{P e i}^{1}$ \\ ${ }^{1}$ Department of Radiation Oncology, The Third Affiliated Hospital of Soochow University, Changzhou 213003, P.R. China \\ ${ }^{2}$ Department of Respiratory, The Seventh People's Hospital of Changzhou, Changzhou 213000, P.R. China \\ *These authors have contributed equally to this work \\ Correspondence to: Zhonghua Ning, email: nzh96086@163.com \\ Wendong Gu, email: hony0428@126.com \\ Honglei Pei, email: hongleipei@126.com \\ Keywords: SII, nasopharyngeal carcinoma, prognosis, PSM \\ Received: April 12, $2017 \quad$ Accepted: June 28, $2017 \quad$ Published: August 02, 2017 \\ Copyright: Jiang et al. This is an open-access article distributed under the terms of the Creative Commons Attribution License 3.0 \\ (CC BY 3.0), which permits unrestricted use, distribution, and reproduction in any medium, provided the original author and source \\ are credited.
}

\section{ABSTRACT}

Systemic immune-inflammation index (SII), based on peripheral lymphocyte, neutrophil, and platelet counts, was recently investigated as a prognostic marker in several tumors. However, SII has not been reported in nasopharyngeal carcinoma (NPC). We evaluated the prognostic value of the SII in 327 patients with NPC. Univariate and multivariate analyses were calculated by the Cox proportional hazards regression model. The time-dependent receiver operating characteristics (ROC) curve was used to compare the discrimination ability for OS. PSM (propensity score matching) was carried out to imbalance the baseline characteristics. Our results showed that SII, PLR, NLR and MLR were all associated with OS in NPC patients in the Kaplan-Meier survival analysis. SII (HR: 2.26; 95\% CI: 1.40-3.66; P=0.001), NLR (HR: 1.66; 95\% CI: 1.08-2.53; $P=0.020$ ), and MLR (HR: 1.99; 95\% CI: 1.17-3.39; $P=0.011$ ) were identified to be the independent prognostic factors. The AUC for SII was bigger than NLR, PLR and MLR for predicting survival in patients with NPC in 3 or 5-years. In the PSM analysis, SII remained an independent predictor for OS in NPC patients $(H R=2.08, C I 1.22-3.55, P=0.007)$. SII is a novel, simple and inexpensive prognostic predictor for patients with NPC. The prognostic value of SII is superior to PLR, NLR and MLR.

\section{INTRODUCTION}

The incidence of nasopharyngeal carcinoma (NPC) has obvious geographic distributions. The high incidence of NPC occurs in southeast of China, Hong Kong and Singapore. There were 60,600 new cases and 34,100 deaths of NPC per year in China [1]. Radiotherapy is the preferred treatment for NPC. Although multidisciplinary treatment based on radiotherapy has achieved good results, local failure and distant metastasis are still more common [2]. Patients with recurrence or distant metastasis had poor prognosis, and five-year overall survival (OS) is less than $30 \%$ [3]. Currently, the gold standard for predicting the prognosis of NPC patients is the American Joint Committee on Cancer tumor-node-metastasis (AJCC TNM) staging system. However, NPC patients at the same TNM stage and received similar therapy usually had different outcomes $[4,5]$. Therefore, it is important to explore the biomarkers which can improve prognostic prediction of NPC. 
Since the first reported by Virchow in 1863 [6], there have been accumulating evidences supporting that inflammation contributes to tumor growth, progression and metastasis [7]. Recently, the systemic inflammatory response biomarkers such as circulating immune cells have been found to be independent markers of prognosis in a variety of cancers. Circulating immune cells mainly included neutrophil, platelet, lymphocyte and monocyte which derived from the peripheral blood. Systemic inflammatory biomarkers such as platelet lymphocyte ratio (PLR), neutrophil lymphocyte ratio (NLR) and monocyte lymphocyte ratio (MLR) have emerged as prognostic markers in a variety of cancer, including NPC [8-10]. These markers only integrate two circulating immune cells. Recently, systemic immune-inflammation index (SII), based on three circulating immune cells (peripheral lymphocyte, neutrophil, and platelet), has proved to be a novel prognostic marker in hepatocellular carcinoma [11-13], esophageal cancer [14], colorectal cancer [15], gastric cancer [16], small cell lung cancer [17] and renal cell cancer [18]. However, the prognostic value of SII has not been reported in NPC. In our study, we first reported the prognostic value of SII in patients with NPC. We also evaluated whether SII has more advantages to predict prognosis of NPC than other systemic inflammatory biomarkers. To increase statistical power and to further elaborate on the possible prognostic impact of SII, both Cox's proportional hazards model analysis as well as propensity score matching (PSM) were applied.

\section{RESULTS}

\section{Baseline clinical and characteristics}

Finally, a total of 327 patients were enrolled in this study. There were 243 males (74.3\%) and 84 females $(25.7 \%)$ with age ranging from 20 to 80 years (median 50 years). Among them, 87 patients' body mass index (BMI) were over 25. According to the TNM stage, there were 12 patients at stage I, 54 patients at stage II, 146 patients at stage III and 115 patients at stage IV. The 3and 5-year overall survival (OS) rates were $76.3 \%$ and $65.7 \%$, respectively. The association between SII, PLR, NLR, MLR and clinicopathological features are shown in Table 1, 2. Patients with SII $>403$ were more likely to be $\mathrm{BMI}>25 \quad(P=0.002)$, advanced $\mathrm{T}$ stage $(P=0.013)$ and advanced TNM stage $(P=0.031)$ (Table 1$)$. We also explored the association between SII, PLR, NLR and MLR (Table 2). It was found that SII was associated with other inflammation-based prognostic indexes (all $P<0.001$ ).

\section{The prognostic significance of SII, NLR, PLR and MLR}

Compared with a lower SII $(\leq 403)$, a higher SII $(>403)$ was associated with significant worse OS in NPC patients $(P<0.001$, Figure 1A). High PLR, NLR and MLR scores were also associated with poor OS $(P=0.038$, $P=0.024, P=0.008$, respectively) (Figure $1 \mathrm{~B}, 1 \mathrm{C}, 1 \mathrm{D}$ ). In the univariate analysis, sex, age, $\mathrm{T}$ stage, $\mathrm{N}$ stage, $\mathrm{SII}$, PLR, NLR and MLR were identified as the significant prognostic indexes (Table 3). We found that SII, PLR, NLR and MLR were high correlation. So, four separate multivariate models (SII, PLR, NLR and MLR) were run to avoid problems with the presence of multicollinearity. In the multivariate analysis, SII (HR: 2.26; 95\% CI: 1.40-3.66; $P=0.001$ ), NLR (HR: 1.66 ; 95\% CI: 1.08 $2.53 ; P=0.020$ ), and MLR (HR: $1.99 ; 95 \% \mathrm{CI}: 1.17-3.39$; $P=0.011$ ) were identified to be the independent prognostic factors, after adjustment for other characteristics (Table 3). Among the four inflammation-based prognostic indexes, only PLR was not an independent risk factor for OS $(P>0.05)$ (Table 3$)$.

To compare the discriminatory ability of SII, PLR, NLR, MLR, we generated ROC curves for the survival status at 3 year and 5 years of follow-up and statistically compared the differences of estimated AUC (Figure 2). The results showed that at the follow-up of 3 year, the AUC value of SII (categorical) was significantly higher than that of the NLR, PLR or MLR. The AUC for SII (categorical) was still higher than NLR, PLR and MLR for predicting survival in patients with NPC in 5-years (Figure 2A, 2B). SII (continuous) showed the same results (Figure $2 \mathrm{C}, 2 \mathrm{D})$. It indicated that SII is superior to NLR, PLR or MLR as a predictive biomarker in NPC patients.

In order to further identify features of patients with better value of SII in the different TNM staging, we performed subgroup survival analysis. It included only the cases with III and IV disease for subgroup analysis because the patient number in I and II stage was small. We observed that in III and IV patients, high SII scores was significantly associated with poor OS $(P=0.043$ for III patients and $P=0.024$ for IV patients) as shown in Figure $3 \mathrm{~A}$ and $3 \mathrm{~B}$.

\section{Propensity score matching analysis}

Considered the BMI, T stage and AJCC TNM stage were imbalance between $\mathrm{SII} \leq 403$ and $\mathrm{SII}>403$ NPC patients which may affect the reliability of the results (Table 1), we applied a 1:1 PSM ratio to minimize these differences. In the PSM analysis, we selected 108 patients from $\mathrm{SII} \leq 403$ group with matched pairings of the 108 SII $>403$ patients. Sex, age, BMI, T stage, N stage and AJCC stage were included for the one-to-one match process. The main characteristics were balanced and evenly distributed between two groups (all $P>0.2$ ) (Table 1). In the matched 216 patients' survival analysis, high SII scores was significantly associated with poor OS $(P=0.011)$ (Figure 4). In addition, the multivariate analyses indicated SII remained an independent predictor for OS in NPC patients $(\mathrm{HR}=2.08$, CI 1.22-3.55, $P=0.007)$ (Table 4). 
Table 1: Baseline characteristics for patients with $\mathrm{SII} \leq 403$ versus $\mathrm{SII}>\mathbf{4 0 3}$ before and after propensity matching

\begin{tabular}{|c|c|c|c|c|c|c|c|c|c|c|}
\hline \multirow[b]{2}{*}{ Clinical parameter } & \multicolumn{5}{|c|}{ Unmatched (complete) dataset } & \multicolumn{5}{|c|}{ Matched (1:1) dataset } \\
\hline & $\begin{array}{c}\mathrm{SII} \leq 403 \\
(123)\end{array}$ & $\begin{array}{c}\text { SII }>403 \\
(204)\end{array}$ & $\chi^{2}$ & $P$ & S.D & $\begin{array}{c}\mathrm{SII} \leq 403 \\
(108)\end{array}$ & $\begin{array}{c}\text { SII }>403 \\
(108)\end{array}$ & $\chi^{2}$ & $\boldsymbol{P}$ & S.D \\
\hline Sex & & & 0.46 & 0.498 & & & & 0.41 & 0.519 & \\
\hline Male & 94 & 149 & & & 0.08 & 85 & 81 & & & 0.09 \\
\hline Female & 29 & 55 & & & 0.08 & 23 & 27 & & & 0.09 \\
\hline Age mean $(\mathrm{SD})$ & $54(11)$ & $52(12)$ & & & 0.17 & $53(12)$ & $52(12)$ & & & 0.08 \\
\hline BMI & & & 10.06 & $0.002^{*}$ & & & & 0.33 & 0.564 & \\
\hline$\leq 25$ & 78 & 162 & & & 0.70 & 70 & 74 & & & 0.08 \\
\hline$>25$ & 45 & 42 & & & 0.70 & 38 & 34 & & & 0.08 \\
\hline T stage & & & 10.78 & $0.013^{*}$ & & & & 1.54 & 0.674 & \\
\hline $\mathrm{T} 1$ & 34 & 27 & & & 0.36 & 23 & 20 & & & 0.07 \\
\hline $\mathrm{T} 2$ & 29 & 57 & & & 0.10 & 27 & 28 & & & 0.01 \\
\hline $\mathrm{T} 3$ & 38 & 71 & & & 0.08 & 35 & 30 & & & 0.10 \\
\hline $\mathrm{T} 4$ & 22 & 49 & & & 0.15 & 23 & 30 & & & 0.15 \\
\hline $\mathrm{N}$ stage & & & 6.51 & 0.089 & & & & 1.77 & 0.625 & \\
\hline No & 20 & 15 & & & 0.28 & 9 & 8 & & & 0.03 \\
\hline N1 & 29 & 57 & & & 0.10 & 29 & 35 & & & 0.12 \\
\hline $\mathrm{N} 2$ & 55 & 99 & & & 0.08 & 51 & 52 & & & 0.02 \\
\hline N3 & 19 & 33 & & & 0.01 & 19 & 13 & & & 0.16 \\
\hline AJCC stage & & & 8.85 & $0.031^{*}$ & & & & 0.00 & 1.000 & \\
\hline I & 9 & 3 & & & 0.29 & 3 & 3 & & & 0 \\
\hline II & 23 & 31 & & & 0.09 & 21 & 21 & & & 0 \\
\hline III & 53 & 93 & & & 0.05 & 47 & 47 & & & 0 \\
\hline IV & 38 & 77 & & & 0.15 & 37 & 37 & & & 0 \\
\hline chemotherapy & & & 3.26 & 0.071 & & & & 0.43 & 0.513 & \\
\hline No & 32 & 36 & & & 0.20 & 26 & 22 & & & 0.09 \\
\hline Yes & 91 & 168 & & & 0.20 & 82 & 86 & & & 0.09 \\
\hline IMRT & & & 1.12 & 0.291 & & & & 0.70 & 0.402 & \\
\hline No & 79 & 119 & & & 0.12 & 69 & 63 & & & 0.11 \\
\hline Yes & 44 & 85 & & & 0.12 & 39 & 45 & & & 0.11 \\
\hline
\end{tabular}

SD: standard deviation; BMI: body mass index; AJCC: American Joint Committee on Cancer; IMRT: intensity-modulated radiotherapy; SII: systemic immune-inflammation index; S.D: standardized difference.

\section{DISCUSSION}

Recently, more and more studies have indicated that inflammation plays an important role in cancer development and metastasis [7]. Pretreatment NLR, PLR and MLR were unfavorable prognostic factors for NPC patients in previous studies [8,10,19-22]. A novel systemic inflammation score-SII, as a prognostic factor for poor survival, was considered to be superior to NLR and PLR in hepatocellular carcinoma [11-13], small cell lung cancer [17], esophageal cancer [14] and gastric cancer [16]. However, the prognostic value of SII has not been reported in NPC. In this study, pretreatment SII was confirmed to be a novel independent prognostic factor for 
Table 2: Relationship between NLR, PLR or MLR and clinicopathological characteristics of patients with nasopharyngeal carcinoma

\begin{tabular}{|c|c|c|c|c|c|c|c|c|c|c|c|c|}
\hline \multirow{2}{*}{$\begin{array}{l}\text { Clinical } \\
\text { parameter }\end{array}$} & \multicolumn{4}{|c|}{ NLR } & \multicolumn{4}{|c|}{ PLR } & \multicolumn{4}{|c|}{ MLR } \\
\hline & $\begin{array}{l}\leq 2.26 \\
(158)\end{array}$ & $\begin{array}{l}>2.26 \\
(169)\end{array}$ & $\chi^{2}$ & $P$ & $\begin{array}{l}\leq 112 \\
(141)\end{array}$ & $\begin{array}{l}>112 \\
(186)\end{array}$ & $\chi^{2}$ & $P$ & $\begin{array}{c}\leq 0.25 \\
(97)\end{array}$ & $\begin{array}{l}>0.25 \\
(230)\end{array}$ & $\chi^{2}$ & $P$ \\
\hline Sex & & & 1.88 & 0.170 & & & 0.93 & 0.334 & & & 0.33 & 0.564 \\
\hline Male & 112 & 131 & & & 101 & 142 & & & 70 & 173 & & \\
\hline Female & 46 & 38 & & & 40 & 44 & & & 27 & 57 & & \\
\hline Age & & & 0.05 & 0.828 & & & 1.31 & 0.288 & & & 0.30 & 0.584 \\
\hline$<50$ & 58 & 64 & & & 48 & 74 & & & 34 & 88 & & \\
\hline$\geq 50$ & 100 & 105 & & & 93 & 112 & & & 63 & 142 & & \\
\hline BMI & & & 2.23 & 0.135 & & & 7.02 & 0.008 & & & 3.88 & 0.049 \\
\hline$\leq 25$ & 110 & 130 & & & 93 & 147 & & & 64 & 176 & & \\
\hline$>25$ & 48 & 39 & & & 48 & 39 & & & 33 & 54 & & \\
\hline T stage & & & 1.06 & 0.787 & & & 0.70 & 0.874 & & & 5.98 & 0.113 \\
\hline $\mathrm{T} 1$ & 30 & 31 & & & 28 & 33 & & & 16 & 45 & & \\
\hline $\mathrm{T} 2$ & 41 & 45 & & & 35 & 51 & & & 31 & 55 & & \\
\hline $\mathrm{T} 3$ & 56 & 53 & & & 49 & 60 & & & 36 & 73 & & \\
\hline $\mathrm{T} 4$ & 31 & 40 & & & 29 & 42 & & & 14 & 57 & & \\
\hline $\mathrm{N}$ stage & & & 4.46 & 0.216 & & & 4.69 & 0.196 & & & 2.89 & 0.409 \\
\hline N0 & 16 & 19 & & & 19 & 16 & & & 13 & 22 & & \\
\hline $\mathrm{N} 1$ & 34 & 52 & & & 30 & 56 & & & 23 & 63 & & \\
\hline $\mathrm{N} 2$ & 79 & 75 & & & 67 & 87 & & & 42 & 112 & & \\
\hline N3 & 29 & 23 & & & 25 & 27 & & & 19 & 33 & & \\
\hline $\begin{array}{l}7^{\text {th }} \text { AJCC TNM } \\
\text { stage }\end{array}$ & & & 2.75 & 0.432 & & & 4.44 & 0.217 & & & 0.491 & 0.921 \\
\hline $\mathrm{I}$ & 5 & 7 & & & 7 & 5 & & & 3 & 9 & & \\
\hline II & 21 & 33 & & & 17 & 37 & & & 17 & 37 & & \\
\hline III & 75 & 71 & & & 66 & 80 & & & 45 & 101 & & \\
\hline IV & 57 & 58 & & & 51 & 64 & & & 32 & 83 & & \\
\hline chemotherapy & & & 0.26 & 0.613 & & & 0.21 & 0.644 & & & 0.298 & 0.585 \\
\hline No & 31 & 37 & & & 31 & 37 & & & 22 & 46 & & \\
\hline Yes & 127 & 132 & & & 110 & 149 & & & 75 & 184 & & \\
\hline IMRT & & & 0.69 & 0.406 & & & 0.69 & 0.408 & & & 0.098 & 0.754 \\
\hline No & 92 & 106 & & & 89 & 109 & & & 60 & 138 & & \\
\hline Yes & 66 & 63 & & & 52 & 77 & & & 37 & 92 & & \\
\hline SII & & & 39.66 & $<0.001^{*}$ & & & 98.08 & $<0.001^{*}$ & & & 43.91 & $<0.001^{*}$ \\
\hline$\leq 403$ & 87 & 36 & & & 96 & 27 & & & 63 & 60 & & \\
\hline$>403$ & 71 & 133 & & & 45 & 159 & & & 34 & 170 & & \\
\hline
\end{tabular}

(Continued) 


\begin{tabular}{|c|c|c|c|c|c|c|c|c|c|c|c|c|}
\hline \multirow{2}{*}{$\begin{array}{l}\text { Clinical } \\
\text { parameter }\end{array}$} & \multicolumn{4}{|c|}{ NLR } & \multicolumn{4}{|c|}{ PLR } & \multicolumn{4}{|c|}{ MLR } \\
\hline & $\begin{array}{l}\leq 2.26 \\
(158)\end{array}$ & $\begin{array}{l}>2.26 \\
(169)\end{array}$ & $\chi^{2}$ & $P$ & $\begin{array}{l}\leq 112 \\
(141)\end{array}$ & $\begin{array}{l}>112 \\
(186)\end{array}$ & $\chi^{2}$ & $P$ & $\begin{array}{c}\leq 0.25 \\
(97)\end{array}$ & $\begin{array}{l}>0.25 \\
(230)\end{array}$ & $\chi^{2}$ & $P$ \\
\hline PLR & & & 155.87 & $<0.001^{*}$ & & & & & & & 34.92 & $<0.001^{*}$ \\
\hline$\leq 112$ & 124 & 17 & & & & & & & 66 & 75 & & \\
\hline$>112$ & 34 & 152 & & & & & & & 31 & 155 & & \\
\hline NLR & & & - & - & & & 155.87 & $<0.001^{*}$ & & & 33.18 & $<0.001^{*}$ \\
\hline$\leq 2.26$ & - & - & & & 124 & 34 & & & 71 & 87 & & \\
\hline$>2.26$ & - & - & & & 17 & 152 & & & 26 & 143 & & \\
\hline MLR & & & 33.18 & $<0.001^{*}$ & & & 34.92 & $<0.001^{*}$ & & & - & - \\
\hline$\leq 0.25$ & 71 & 26 & & & 66 & 31 & & & - & - & & \\
\hline$>0.25$ & 87 & 143 & & & 75 & 155 & & & - & - & & \\
\hline
\end{tabular}

BMI: body mass index; AJCC: American Joint Committee on Cancer; IMRT: intensity-modulated radiotherapy; SII: systemic immune-inflammation index; PLR: platelet lymphocyte ratio; NLR: neutrophil lymphocyte ratio; MLR: monocyte lymphocyte ratio.

patients with NPC. The prognostic value of SII is greater than NLR, PLR and MLR in NPC patients. In addition, SII was significantly correlated with OS in different TNM stage subgroup. Compared with other prognostic factors, SII based on standard laboratory measurements of total platelet, neutrophil, and lymphocyte counts is simple, noninvasive and low cost in clinical practice. Thus, there
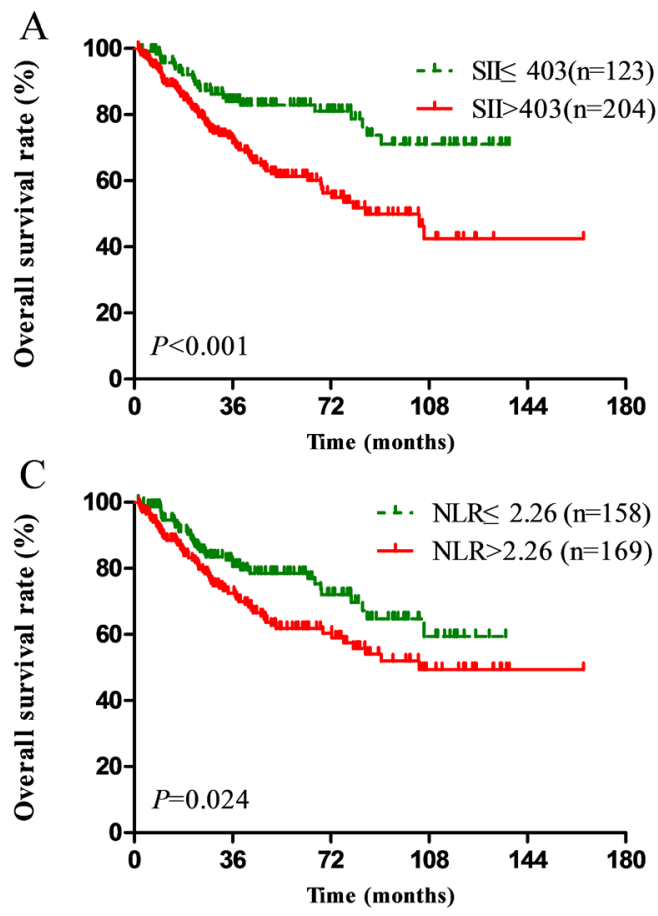

is a potential for SII to be used as a marker for prognosis and treatment response surveillance.

Several potential mechanisms may explain our results. First, neutrophils, as a type of inflammatory cells, are involved in different steps of tumor development through the production of a variety of cytokines, such as oncostatin M, interleukin-6, hepatocyte growth factor, and
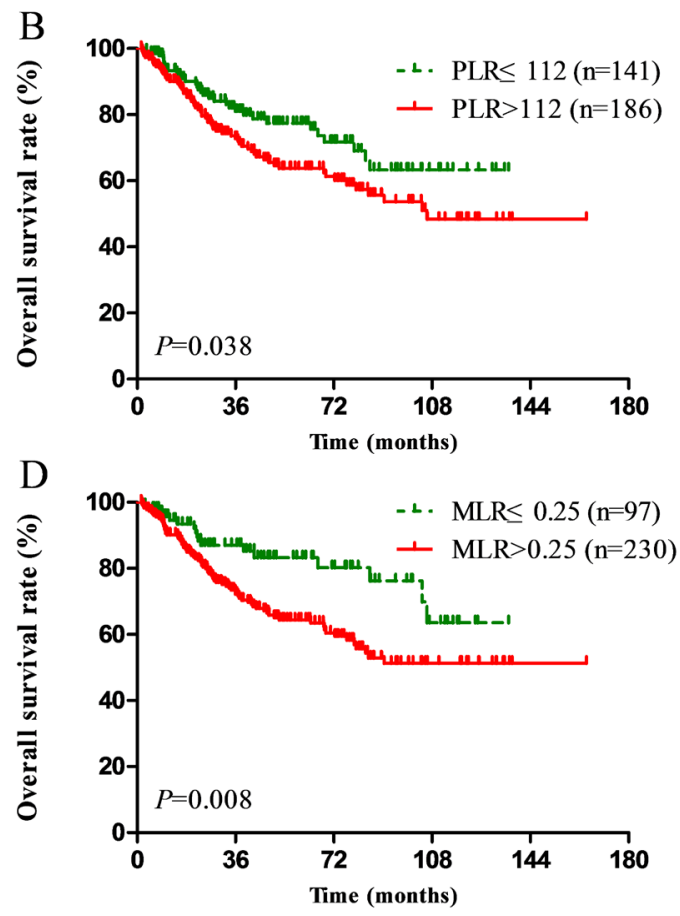

Figure 1: Kaplan-Meier survival curves for patients stratified based on (A) SII, (B) PLR, (C) NLR and (D) MLR in unmatched complete datasets. 
Table 3: Univariate and multivariate cox regression analyses for overall survival in patients with nasopharyngeal carcinoma (unmatched complete datasets)

\begin{tabular}{|c|c|c|c|c|}
\hline \multirow{2}{*}{ Variables } & \multicolumn{2}{|c|}{ Univariate analysis } & \multicolumn{2}{|c|}{ Multivariate analysis } \\
\hline & HR $(95 \% C I)$ & $P$ value & HR $(95 \% C I)$ & $P$ value \\
\hline \multicolumn{5}{|l|}{ Sex } \\
\hline Female vs. Male & $0.39(0.22-0.69)$ & $0.001^{*}$ & $0.40(0.23-0.72)$ & $0.002^{*}$ \\
\hline \multicolumn{5}{|l|}{ Age } \\
\hline$\geq 50$ vs. $<50$ & $1.63(1.05-2.51)$ & $0.028^{*}$ & $1.85(1.18-2.88)$ & $0.007^{*}$ \\
\hline \multicolumn{5}{|l|}{ BMI } \\
\hline$>25$ vs. $\leq 25$ & $0.75(0.46-1.24)$ & 0.264 & & \\
\hline T stage & & $0.009^{*}$ & & $0.039^{*}$ \\
\hline $\mathrm{T} 1$ & Ref. & & Ref. & \\
\hline $\mathrm{T} 2$ & $1.77(0.87-3.61)$ & 0.114 & $1.67(0.81-3.44)$ & 0.167 \\
\hline $\mathrm{T} 3$ & $2.39(1.21-4.72)$ & $0.012^{*}$ & $2.11(1.06-4.21)$ & $0.034^{*}$ \\
\hline $\mathrm{T} 4$ & $3.199(1.57-6.51)$ & $0.001^{*}$ & $2.75(1.34-5.66)$ & $0.006^{*}$ \\
\hline $\mathrm{N}$ stage & & $0.027^{*}$ & & $0.031^{*}$ \\
\hline No & Ref. & & Ref. & \\
\hline N1 & $2.34(0.97-5.64)$ & 0.060 & $2.24(0.92-5.44)$ & 0.075 \\
\hline $\mathrm{N} 2$ & $2.83(1.20-6.65)$ & 0.017 & $2.92(1.23-6.94)$ & $0.015^{*}$ \\
\hline N3 & $4.07(1.59-10.41)$ & 0.003 & $4.04(1.54-10.61)$ & $0.005^{*}$ \\
\hline \multicolumn{5}{|l|}{ chemotherapy } \\
\hline YES vs. NO & $1.11(0.70-1.76)$ & 0.670 & & \\
\hline \multicolumn{5}{|l|}{ IMRT } \\
\hline YES vs. NO & $0.79(0.51-1.24)$ & 0.310 & & \\
\hline \multicolumn{5}{|l|}{ SII } \\
\hline$>403$ vs. $\leq 403$ & $2.34(1.46-3.74)$ & $<0.001^{*}$ & $2.26(1.40-3.66)$ & $0.001^{* a}$ \\
\hline \multicolumn{5}{|l|}{ NLR } \\
\hline$>2.26$ vs. $\leq 2.26$ & $1.58(1.04-2.38)$ & $0.031^{*}$ & $1.66(1.08-2.53)$ & $0.020^{* b}$ \\
\hline \multicolumn{5}{|l|}{ PLR } \\
\hline$>112$ vs. $\leq 112$ & $1.54(1.01-2.35)$ & $0.047^{*}$ & $1.54(0.99-2.38)$ & $0.051^{\mathrm{c}}$ \\
\hline \multicolumn{5}{|l|}{ MLR } \\
\hline$>0.25$ vs. $\leq 0.25$ & $1.98(1.17-3.35)$ & $0.011^{*}$ & $1.99(1.17-3.39)$ & $0.011^{* d}$ \\
\hline
\end{tabular}

BMI: body mass index; IMRT: intensity-modulated radiotherapy; SII: systemic immune-inflammation index; PLR: platelet lymphocyte ratio; NLR: neutrophil lymphocyte ratio; MLR: monocyte lymphocyte ratio; HR: hazard ratio; CI: confidence interval; Ref: reference. ${ }^{a}$ The variables (sex, age, T stage, N stage and SII) were tested in a multivariate analysis. ${ }^{\mathrm{b}}$ The variables (sex, age, T stage, $\mathrm{N}$ stage and NLR) were tested in a multivariate analysis. ${ }^{\mathrm{c}}$ The variables (sex, age, $\mathrm{T}$ stage, $\mathrm{N}$ stag and PLR) were tested in a multivariate analysis. ${ }^{\mathrm{d}}$ The variables (sex, age, $\mathrm{T}$ stage, $\mathrm{N}$ stag and MLR) were tested in a multivariate analysis. 

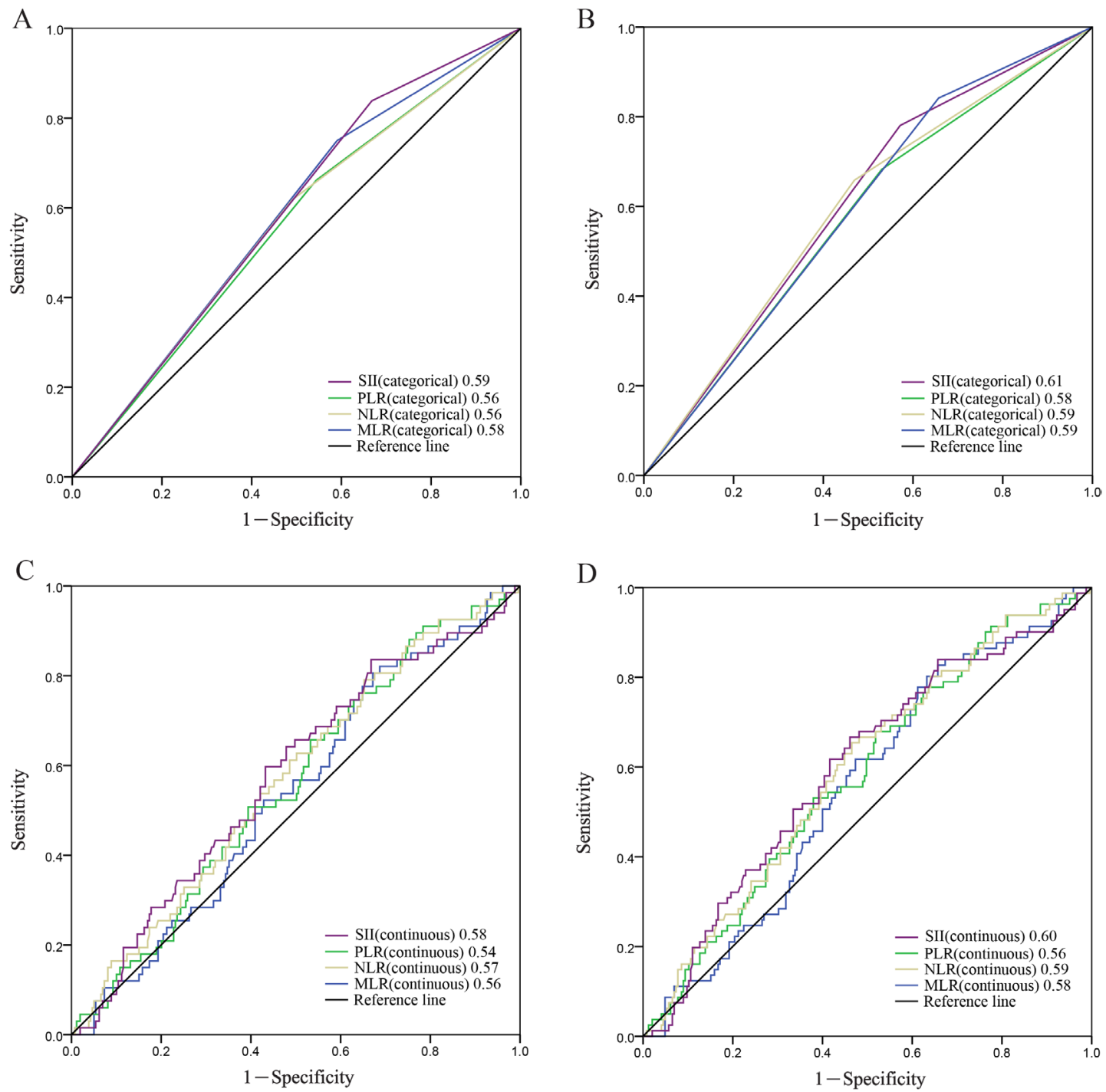

Figure 2: Predictive ability of the SII (categorical) was compared with PLR(categorical), NLR(categorical) and MLR(categorical) by ROC curves in 3-years (A) and 5-years (B). Predictive ability of the SII (continuous) was compared with PLR(continuous), NLR(continuous) and MLR(continuous) by ROC curves in 3-years (C) and 5-years (D).

A

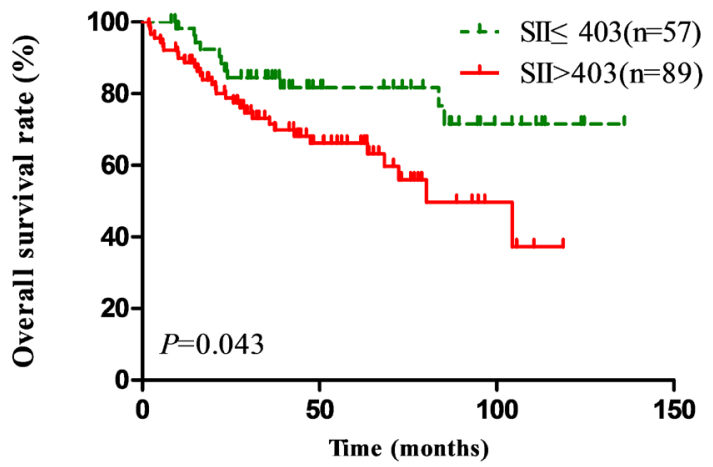

B

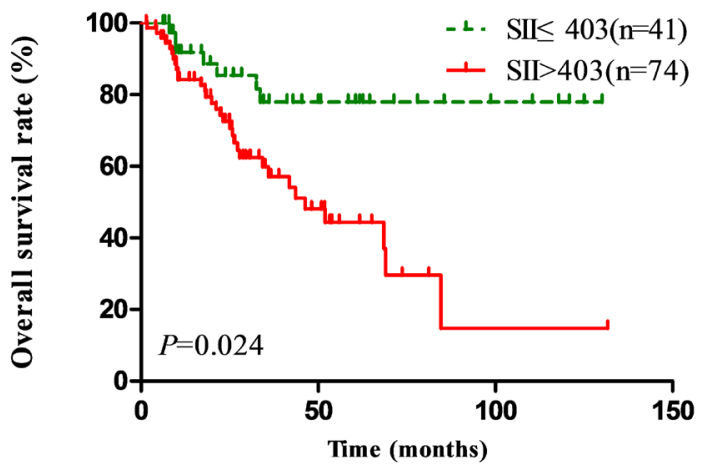

Figure 3: Effect of the SII on the survival of nasopharyngeal carcinoma patients in III stage (A), and IV stage (B). 
tumor necrosis factor $[23,24]$. It can enhance the invasion, proliferation, and metastasis of cancer cells as well as aid them to evade immune surveillance $[25,26]$. The elevated neutrophils release plenty of reactive oxygen species and nitric oxide. They can lead to $\mathrm{T}$ cell activation disorders [27]. Second, platelets can direct contact with circulating tumor cells. It promotes tumor cell extravasation to metastatic sites [28, 29]. In addition, increased circulating platelets and neutrophils produce vascular endothelial growth factor, angiopoietin-1, and fibroblast growth factor-2, causing tumor angiogenesis. [11, 30, 31]. Third, cytotoxic lymphocytes play a fundamental role in cell-mediated immunologic destruction of cancer cells. Circulating lymphocytes can secrete several cytokines, such as IFN- $\gamma$ and TNF- $\alpha$, to control tumor growth and improve prognosis of cancer patients [32], and the decreased lymphocyte count and function cannot be responsible for immune surveillance to remove tumor cells [25, 32]. Fourth, Monocytes release monocyte chemoattractant protein-1 to stimulate and mediate tumorassociated monocyte infiltration in solid tumors and then produce various chemokines, such as TGF- $\alpha$, TNF- $\alpha$, IL-1, and IL-6 which promote tumorigenesis, angiogenesis, and distant metastasis of malignant tumors [33].

In recent years, several studies showed that systemic inflammatory scores have emerged as prognostic markers in NPC. He et al. reported pretreatment NLR and percentages of lymphocyte and neutrophil are independent prognostic factors and may serve as clinically convenient and useful biomarkers for survival of patients with NPC [19]. Sun et al. reported that pretreatment NLR and PLR can be independent prognostic factors for patients with NPC [20]. Jiang et al. reported elevated PLR values were associated with poor overall survival, cancer-specific survival, and distant metastasis-free survival for patients with NPC [22]. Lu et al. reported the pretreatment NLR was an independent prognostic factor in NPC, and NLR, LMR, and PLR might be a useful complement to TNM staging in the prognostic assessment of NPC patients [21]. In the subsequent meta-analysis, $\mathrm{Su}$ et al. found NLR and lymphocyte counts were the 2 most reported prognostic predictors for patients with NPC [10]. However, no study evaluated the prognostic value of SII. SII was based on three circulating immune cells, while NLR, PLR and MLR was based on two circulating immune cells. SII should be a more objective marker that reflects the balance between host inflammatory and immune response status than all the other systemic inflammation scores. In fact, our results confirmed that SII is indeed superior to PLR, NLR and MLR.

Although our results are valuable in NPC, there are some limitations in our study. First, our study was conducted retrospectively in a single center, and the prognostic value of the SII was not verified in a validation cohort. However, we used PSM analysis which can minimize group differences in the baseline characteristics. Second, there was heterogeneity in the patient treatment, thus it was hard to analyze the impact of the SII on patients' outcome in different treatment patterns. Third, EBV-DNA was one of the important risk factors of distant metastasis. It was not included in this analysis mainly because EBV-DNA was not performed as a routine clinical practice in our hospital before 2008. Forth, due to the absence of some data, we cannot classify all patients according to the 8th AJCC stage. Finally, some diseases such as diabetes, rheumatic diseases and autoimmune disease, may also affect circulating immune cells, which may influence the SII score.

In conclusion, pretreatment SII is a novel independent prognostic predictor for patients with NPC.

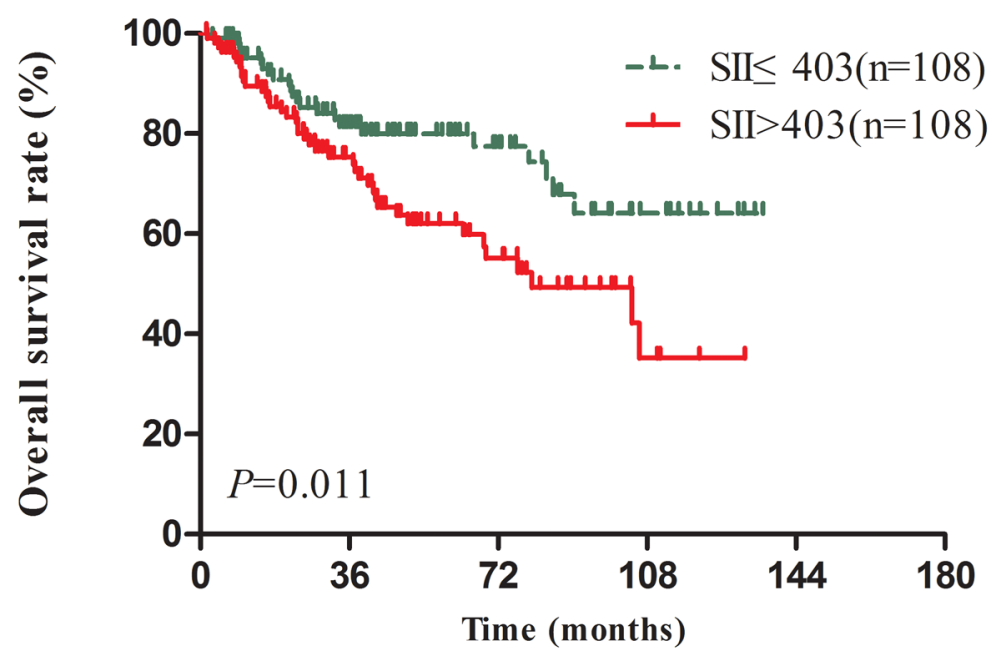

Figure 4: Kaplan-Meier-estimated overall survival distributions from matched datasets for $\mathrm{SII} \leq \mathbf{4 0 3}$ versus $\mathrm{SII}>403$. 
Table 4: Univariate and multivariate cox regression analyses for overall survival in patients with nasopharyngeal carcinoma (matched datasets, $1: 1$ )

\begin{tabular}{|c|c|c|c|c|}
\hline \multirow{2}{*}{ Variables } & \multicolumn{2}{|c|}{ Univariate analysis } & \multicolumn{2}{|c|}{ Multivariate analysis } \\
\hline & HR $(95 \% C I)$ & $P$ value & HR $(95 \% C I)$ & $P$ value \\
\hline \multicolumn{5}{|l|}{ Sex } \\
\hline Female vs. Male & $0.49(0.25-0.93)$ & $0.030^{*}$ & $0.38(0.19-0.74)$ & $0.005^{*}$ \\
\hline \multicolumn{5}{|l|}{ Age } \\
\hline$\geq 50$ vs. $<50$ & $1.54(0.90-2.64)$ & 0.120 & & \\
\hline \multicolumn{5}{|l|}{ BMI } \\
\hline$>25$ vs. $\leq 25$ & $1.02(0.59-1.76)$ & 0.944 & & \\
\hline $\mathrm{T}$ stage & & $0.013^{*}$ & & $0.015^{*}$ \\
\hline $\mathrm{T} 1$ & Ref. & & Ref. & \\
\hline $\mathrm{T} 2$ & $2.50(0.99-6.35)$ & 0.053 & $2.21(0.85-5.73)$ & 0.103 \\
\hline $\mathrm{T} 3$ & $3.25(1.30-8.11)$ & $0.012^{*}$ & $3.38(1.33-8.60)$ & $0.011^{*}$ \\
\hline $\mathrm{T} 4$ & $4.49(1.78-11.31)$ & $0.001^{*}$ & $4.12(1.62-10.48)$ & $0.003^{*}$ \\
\hline $\mathrm{N}$ stage & & $0.040^{*}$ & & 0.066 \\
\hline N0 & Ref. & & Ref. & \\
\hline N1 & $1.52(0.91-10.10)$ & 0.071 & $3.08(0.91-10.51)$ & 0.072 \\
\hline $\mathrm{N} 2$ & $3.57(1.08-11.79)$ & $0.037^{*}$ & $3.57(1.06-12.00)$ & $0.040^{*}$ \\
\hline N3 & $6.59(1.75-24.83)$ & $0.005^{*}$ & $6.12(1.58-23.64)$ & $0.009^{*}$ \\
\hline \multicolumn{5}{|l|}{ chemotherapy } \\
\hline YES vs. NO & $1.09(0.62-1.63)$ & 0.774 & & \\
\hline \multicolumn{5}{|l|}{ IMRT } \\
\hline YES vs. NO & $0.86(0.49-1.51)$ & 0.608 & & \\
\hline \multicolumn{5}{|l|}{ SII } \\
\hline$>403$ vs. $\leq 403$ & $1.91(1.15-3.20)$ & $0.013^{*}$ & $2.08(1.22-3.55)$ & $0.007^{*}$ \\
\hline
\end{tabular}

BMI: body mass index; IMRT: intensity-modulated radiotherapy; SII: systemic immune-inflammation index; HR: hazard ratio; CI: confidence interval; Ref: reference.

The prognostic value of SII is superior to PLR, NLR and MLR in NPC. Based on low cost and easy determination of a full blood count, SII will be a potential marker for NPC prognosis and treatment response surveillance.

\section{MATERIALS AND METHODS}

\section{Patients}

From January 2004 to December 2012, newly identified NPC patients who enrolled in the Third Affiliated Hospital of Soochow University were studied. The inclusion criteria were: (1) NPC was confirmed by histopathology, (2) Karnofsky performance score (KPS) $\geq 70$, (3) patient had detailed medical records, including MRT, CT, and bone scan for staging, (4) patients received radiotherapy for the first time, (5) patients had measurement of neutrophil, platelet, lymphocyte and monocyte at the same time within 1 weeks before therapy. The exclusion criteria were: (1) distant metastases before or during radiotherapy, (2) radiotherapy uncompleted, (3) any severe coexisting disease mainly including severe dysfunction of heart, lung, liver, or kidney, (4) signs of infection such as acute pancreatitis, cholangitis, or other active concomitant infections. At last, 327 patients were enrolled in this study. This study was undertaken according to the Declaration of Helsinki and was approved by the Ethics Committee of Third Affiliated Hospital of Soochow University. Written informed consent was obtained from all patients. 
327 patients were treated with continuously definitive radiotherapy with daily fractions of $2.0 \mathrm{~Gy}$ and five fractions per week by 6-8 MV x-ray. Among them, 198 patients with NPC were treated with 2-dimensional radiotherapy (2DRT), and 129 were treated with intensitymodulated radiotherapy (IMRT). The primary tumor was given a total dose of 60-78 Gy in 2DRT. In IMRT, the radiation dose-ranges to the nasopharynx, lymph node-positive area and lymph node-negative area were $60-80,60-70$ and 50-56 Gy, respectively. The regimen of inductive chemotherapy was one or two cycles of paclitaxel $\left(135 \mathrm{mg} / \mathrm{m}^{2}\right)$ and nedaplatin $\left(80 \mathrm{mg} / \mathrm{m}^{2}\right)$ every 3 weeks. Two to three cycles of nedaplatin $\left(80 \mathrm{mg} / \mathrm{m}^{2}\right)$ every 3 weeks were used in concurrent chemoradiotherapy. A total of 259 (79.2\%) patients received chemotherapy, with 36 patients treated with inductive chemotherapy only, 69 patients treated with concurrent chemotherapy and 154 patients treated with inductive chemotherapy plus concurrent chemotherapy.

All peripheral blood was collected and tested for neutrophils, lymphocytes, platelet, and monocyte counts within 1 weeks before therapy. The definitions of SII, PLR, NLR and MLR are described as follows: $\mathrm{SII}=$ platelet*neutrophil/lymphocyte; $\mathrm{PLR}=$ platelet $/$ lymphocyte; NLR= neutrophil/lymphocyte; MLR= monocyte/lymphocyte. The optimal cutoff values including SII (SII $\leq 403$, SII $>403)$, NLR $(\mathrm{NLR} \leq 2.26$, NLR $>2.26)$, PLR $(P L R \leq 112, \quad P L R>112)$ and MLR (MLR $\leq 0.25$, MLR $>0.25$ ) were determined by using $X$-tile software (http://www.tissuearray.org/rimmlab) [34].

\section{Follow-up}

All patients were followed up every three months in the first 2 years, every six months until 5 years, and then once annually. The latest follow-up was conducted at the end of August 2015. All patients were followed up by phone calls and regular letters. The observation time in this study was the interval from the date of diagnosis to death or latest follow-up. Survived patients were censored on the day of the last follow-up. The median follow-up was 38.3 months (range, 2 to 164.6 months).

\section{Statistical analysis}

The correlations between SII, PLR, NLR and MLR and clinicopathological factors were analyzed by the $\chi^{2}$ test. Pearson correlation analyses were applied to analyze the correlation among SII, PLR, NLR and MLR. Survival curves were plotted using the KaplanMeier method and compared using the log-rank test. Univariate and multivariate analyses were calculated by the Cox proportional hazards regression model. The time-dependent receiver operating characteristics (ROC) curve was used to compare the discrimination ability for OS. PSM was carried out because of imbalance in the baseline characteristics. PSM was done with a nearestneighbour matching algorithm, allowing a maximum tolerated difference between propensity scores less than $30 \%$ of the propensity score SD. Standardised group differences were calculated as the means divided by the square root of the half sum of the two variances [35]. PSM was carried out using SPSS 22.0 (SPSS, Chicago, IL) with Statistics Regression and Python Essentials. Statistical analysis was conducted with SPSS 22.0 (SPSS, Chicago, IL), and Graphpad Prism 6.01 (La Jolla, CA, USA). A $P$ value less than 0.05 was considered to indicate statistical significance.

\section{CONFLICTS OF INTEREST}

The authors disclose no potential conflicts of interest.

\section{REFERENCES}

1. Chen W, Zheng R, Baade PD, Zhang S, Zeng H, Bray F, Jemal A, Yu XQ, He J. Cancer statistics in China, 2015. CA Cancer J Clin. 2016; 66:115-132.

2. Yeh SA, Tang Y, Lui CC, Huang YJ, Huang EY. Treatment outcomes and late complications of 849 patients with nasopharyngeal carcinoma treated with radiotherapy alone. Int J Radiat Oncol Biol Phys. 2005; 62:672-679.

3. Smee RI, Meagher NS, Broadley K, Ho T, Williams JR, Bridger GP. Recurrent nasopharyngeal carcinoma: current management approaches. Am J Clin Oncol. 2010; 33:469-473.

4. Wang HY, Sun BY, Zhu ZH, Chang ET, To KF, Hwang JS, Jiang H, Kam MK, Chen G, Cheah SL, Lee M, Liu ZW, Chen J, et al. Eight-signature classifier for prediction of nasopharyngeal [corrected] carcinoma survival. J Clin Oncol. 2011; 29:4516-4525.

5. Wei WI, Sham JS. Nasopharyngeal carcinoma. Lancet. 2005; 365:2041-2054.

6. Balkwill F, Mantovani A. Inflammation and cancer: back to Virchow? Lancet. 2001; 357:539-545.

7. Hanahan D, Weinberg RA. Hallmarks of cancer: the next generation. Cell. 2011; 144:646-674.

8. Li XH, Chang H, Xu BQ, Tao YL, Gao J, Chen C, Qu C, Zhou S, Liu SR, Wang XH, Zhang WW, Yang X, Zhou SL, Xia YF. An inflammatory biomarker-based nomogram to predict prognosis of patients with nasopharyngeal carcinoma: an analysis of a prospective study. Cancer Med. 2017; 6:310-319.

9. Chua ML, Tan SH, Kusumawidjaja G, Shwe MT, Cheah SL, Fong KW, Soong YL, Wee JT, Tan TW. Neutrophilto-lymphocyte ratio as a prognostic marker in locally advanced nasopharyngeal carcinoma: a pooled analysis of two randomised controlled trials. Eur J Cancer. 2016; 67:119-129. 
10. Su L, Zhang M, Zhang W, Cai C, Hong J. Pretreatment hematologic markers as prognostic factors in patients with nasopharyngeal carcinoma: a systematic review and metaanalysis. Medicine (Baltimore). 2017; 96:e6364.

11. Hu B, Yang XR, Xu Y, Sun YF, Sun C, Guo W, Zhang X, Wang WM, Qiu SJ, Zhou J, Fan J. Systemic immuneinflammation index predicts prognosis of patients after curative resection for hepatocellular carcinoma. Clin Cancer Res. 2014; 20:6212-6222.

12. Yang Z, Zhang J, Lu Y, Xu Q, Tang B, Wang Q, Zhang W, Chen S, Lu L, Chen X. Aspartate aminotransferaselymphocyte ratio index and systemic immuneinflammation index predict overall survival in HBV-related hepatocellular carcinoma patients after transcatheter arterial chemoembolization. Oncotarget. 2015; 6:43090-43098. https://doi.org/10.18632/oncotarget.5719.

13. Casadei Gardini A, Scarpi E, Faloppi L, Scartozzi M, Silvestris N, Santini D, de Stefano G, Marisi G, Negri FV, Foschi FG, Valgiusti M, Ercolani G, Frassineti GL. Immune inflammation indicators and implication for immune modulation strategies in advanced hepatocellular carcinoma patients receiving sorafenib. Oncotarget. 2016; 7:67142-67149. https://doi.org/10.18632/oncotarget.11565.

14. Geng Y, Shao Y, Zhu D, Zheng X, Zhou Q, Zhou W, Ni $\mathrm{X}, \mathrm{Wu} \mathrm{C}$, Jiang J. Systemic immune-inflammation index predicts prognosis of patients with esophageal squamous cell carcinoma: a propensity score-matched analysis. Sci Rep. 2016; 6:39482.

15. Passardi A, Scarpi E, Cavanna L, Dall'Agata M, Tassinari D, Leo S, Bernardini I, Gelsomino F, Tamberi S, Brandes AA, Tenti E, Vespignani R, Frassineti GL, et al. Inflammatory indexes as predictors of prognosis and bevacizumab efficacy in patients with metastatic colorectal cancer. Oncotarget. 2016; 7:33210-33219. https://doi.org/10.18632/ oncotarget. 8901 .

16. Huang L, Liu S, Lei Y, Wang K, Xu M, Chen Y, Liu B, Fu Q, Zhang P, Qin K, Cai Y, Fu S, Ge S, Yuan X. Systemic immune-inflammation index, thymidine phosphorylase and survival of localized gastric cancer patients after curative resection. Oncotarget. 2016; 7:44185-44193. https://doi. org/10.18632/oncotarget.9923.

17. Hong X, Cui B, Wang M, Yang Z, Wang L, Xu Q. Systemic immune-inflammation index, based on platelet counts and neutrophil-lymphocyte ratio, is useful for predicting prognosis in small cell lung cancer. Tohoku J Exp Med. 2015; 236:297-304.

18. Lolli C, Basso U, Derosa L, Scarpi E, Sava T, Santoni M, Crabb SJ, Massari F, Aieta M, Conteduca V, Maruzzo M, La Russa F, Wheater M. Systemic immuneinflammation index predicts the clinical outcome in patients with metastatic renal cell cancer treated with sunitinib. Oncotarget. 2016; 7:54564-54571. https://doi. org/10.18632/oncotarget.9923.
19. He JR, Shen GP, Ren ZF, Qin H, Cui C, Zhang Y, Zeng YX, Jia WH. Pretreatment levels of peripheral neutrophils and lymphocytes as independent prognostic factors in patients with nasopharyngeal carcinoma. Head Neck. 2012; 34:1769-1776.

20. Sun W, Zhang L, Luo M, Hu G, Mei Q, Liu D, Long G. Pretreatment hematologic markers as prognostic factors in patients with nasopharyngeal carcinoma: Neutrophillymphocyte ratio and platelet-lymphocyte ratio. Head Neck. 2016; 38:E1332-1340.

21. Lu A, Li H, Zheng Y, Tang M, Li J, Wu H, Zhong W, Gao J, Ou N, Cai Y. Prognostic significance of neutrophil to lymphocyte ratio, lymphocyte to monocyte ratio, and platelet to lymphocyte ratio in patients with nasopharyngeal carcinoma. Biomed Res Int. 2017; 2017:3047802.

22. Jiang R, Zou X, Hu W, Fan YY, Yan Y, Zhang MX, You R, Sun R, Luo DH, Chen QY, Huang PY, Hua YJ, Guo L, Chen MY. The elevated pretreatment platelet-to-lymphocyte ratio predicts poor outcome in nasopharyngeal carcinoma patients. Tumour Biol. 2015; 36:7775-7787.

23. Tecchio C, Scapini P, Pizzolo G, Cassatella MA. On the cytokines produced by human neutrophils in tumors. Semin Cancer Biol. 2013; 23:159-170.

24. Kuper H, Adami HO, Trichopoulos D. Infections as a major preventable cause of human cancer. J Intern Med. 2000; 248:171-183.

25. Mantovani A, Allavena P, Sica A, Balkwill F. Cancer-related inflammation. Nature. 2008; 454:436-444.

26. Diakos CI, Charles KA, McMillan DC, Clarke SJ. Cancerrelated inflammation and treatment effectiveness. Lancet Oncol. 2014; 15:e493-503.

27. Muller I, Munder M, Kropf P, Hansch GM. Polymorphonuclear neutrophils and T lymphocytes: strange bedfellows or brothers in arms? Trends Immunol. 2009; 30:522-530.

28. Labelle M, Begum S, Hynes RO. Direct signaling between platelets and cancer cells induces an epithelialmesenchymal-like transition and promotes metastasis. Cancer Cell. 2011; 20:576-590.

29. Schumacher D, Strilic B, Sivaraj KK, Wettschureck N, Offermanns S. Platelet-derived nucleotides promote tumorcell transendothelial migration and metastasis via P2Y2 receptor. Cancer Cell. 2013; 24:130-137.

30. Cools-Lartigue J, Spicer J, McDonald B, Gowing S, Chow S, Giannias B, Bourdeau F, Kubes P, Ferri L. Neutrophil extracellular traps sequester circulating tumor cells and promote metastasis. J Clin Invest. 2013.

31. Kusumanto YH, Dam WA, Hospers GA, Meijer C, Mulder NH. Platelets and granulocytes, in particular the neutrophils, form important compartments for circulating vascular endothelial growth factor. Angiogenesis. 2003; 6:283-287. 
32. Ferrone C, Dranoff G. Dual roles for immunity in gastrointestinal cancers. J Clin Oncol. 2010; 28:4045-4051.

33. Liao Q, Guo X, Li X, Chen P, Liang F, Tang H, Deng M, Wu M, Ma J, Xiong W, Li G. Analysis of the contribution of nasopharyngeal epithelial cancer cells to the induction of a local inflammatory response. J Cancer Res Clin Oncol. 2012; 138:57-64.
34. Camp RL, Dolled-Filhart M, Rimm DL. X-tile: a new bioinformatics tool for biomarker assessment and outcomebased cut-point optimization. Clin Cancer Res. 2004; 10:7252-7259.

35. D'Agostino RB Jr. Propensity score methods for bias reduction in the comparison of a treatment to a non-randomized control group. Stat Med. 1998; 17:2265-2281. 